\title{
LE CORPS NUMÉRIQUE
}

\author{
Sophie PARICARD

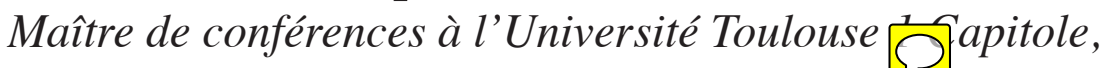 \\ Institut de droit privé CUFR J.-F Champollion, ALBI
}

La réalité numérique a déjà habitué le juriste à penser la personne projetée dans un monde virtuel ${ }^{1}$. Le corps numérique l'invite désormais à concevoir aux côtés du corps concret de chair et d'os, un corps virtuel.

Kantorowicz avait déjà développé cette idée d'un corps abstrait en théorisant les deux corps du Roi ${ }^{2}$. Mais cette projection purement intellectuelle est devenue réalité numérique depuis le décodage du génome ${ }^{3}$ opéré pour la première fois en 2003. C'est ainsi qu'est techniquement apparu le corps numérique constitué

${ }^{1}$ L'usage d'internet est en effet l'occasion d'une formidable et dangereuse collecte d'informations sur la personne de l'internaute. Le big data est le terme autorisé pour caractériser ce nouveau phénomène. Comme l'exprimait A. Levis, "si vous ne payez pas un service sur le Net, c'est que vous n'êtes pas le consommateur, vous êtes le produit vendu”, cité par J-E RAY, “Actualités des TICS”, Dr. Soc. 2011, n 9-10, p. 933. Pour une présentation de l'ensembles du dispositif juridique protégeant actuellement la personne, Voir L. Grynbaum, C. Le Goffic, L. Haidara Morlet, Le droit des activités numériques, Dalloz, Précis, 2014, p. 717 et s. Le Conseil de l'Europe a lancé, le 16 avril 2014, un Guide des droits de l'homme pour les utilisateurs d'internet, lequel figure en annexe de la Recommandation adoptée par le Comité des ministres ce même jour, à destination des Etats membres. Ce guide repose sur l'idée que les droits de l'homme et les libertés fondamentales ont la même valeur dans le monde virtuel que dans le monde réel.

${ }^{2}$ E. Kantorowicz, Les Deux Corps du Roi, NRF, Gallimard, 1957.

${ }^{3}$ Le génome est l'ensemble de l'information génétique (les gènes et autres séquences ADN) contenue dans les chromosomes d'un individu, Commission de l'éthique en sciences et en technologie, 2012, Québec p. 9. 
d'une information cruciale et inédite, l'ensemble des données génétiques de la personne. Les relevés biométriques à partir de l'iris, la voix, de la forme, de la main n'y sont en revanche pas assimilés en ce qu'ils permettent seulement, jusqu'à présent, d'identifier la personne parmi d'autres sans refléter sa vérité ${ }^{4}$

La plus grande part de ses données génétiques sont héréditaires et stables mais certaines, les variants, ne le sont pas. Ce sont les mutations somatiques que l'on trouve notamment dans les cellules cancéreuses ${ }^{5}$ qui permettent aujourd'hui de distinguer différentes formes d'un même cancer quel que soit l'organe touché. Le corps numérique rassemble dès lors des éléments comme le génome bien entendu, mais de façon plus spécifique les gènes dits de prédisposition ou de susceptibilité à certaines pathologies, ou bien encore le génotypage d'une tumeur ${ }^{6}$.

Cette appréhension des données individualisées n'est pas nouvelle dans son principe même. Le meilleur exemple en est certainement la prise en compte de

\begin{abstract}
${ }^{4}$ Voir infra pour plus de développements.
5 "Chaque cellule du corps contient un exemplaire de l'ADN de l'individu : cette information dicte à sa cellule la manière dont elle se comporte et évolue. Les cellules du corps se régénère en deux cellules identiques après avoir fait un duplicata de leur contenu, y compris du matériel génétique. Or il arrive qu'une erreur survienne dans la réplication de l'ADN. La cellule qui en résulte contient alors des caractéristiques génétiques différentes de celle du génotypage stable de l'individu. Il s'agit d'une cellule mutante. Si celle-ci continue de se répliquer, les cellules qui en descendent contiendront toutes la même mutation et pourront évoluer en tumeur puis en cancer", Commission de l'éthique en sciences et en technologie, 2012, Québec, p. 12.
\end{abstract}

${ }^{6}$ Cette expression a pu être tirée de l'expérience suivante. Un patient était atteint d'un cancer du pancréas avec des métastases résistantes à la chimiothérapie utilisée dans ce type de cancer. Des médecins ont alors implanté une partie de sa tumeur dans plusieurs cohortes de souris, créant ainsi des clones du malade et testant sur eux plusieurs traitements. L'un des médicaments fonctionnant bien fut administré au patient qui guérit de son cancer, alors que ce cancer est mortel dans la plupart des cas. "L'utilisation d'un avatar personnalisé de cancer offre la possibilité d'essayer plusieurs combinaisons et même de faire des erreurs avant de commencer un traitement. C'est la direction que prennent plusieurs équipes de recherche. Cette technique du double biologique ne se limite pas au cancer elle est exploitée pour analyser in vivo le cas de personnes souffrant ou risquant de souffrir de diabète de type 1 selon le professeur Philippe Froguel (...) Cette forme de modélisation a été décrite aux rapporteurs lors de leur visite à Gustave RoussY", Office Parlementaire d'Evaluation des Choix Scientifiques et Technologiques (OPECST), A. Claeys, J-S Viallatte, Rapport provisoire, "Les progrès de la génétique, vers une médecine de précision ? Les enjeux scientifiques, technologiques, sociaux et éthiques de la médecine personnalisée", janvier 2014, p. 47. 
l'histocompatibilité tissulaire dans le don d'organes ou de cellules suite notamment aux travaux de Jean Dausset. Mais, les questions qui émergent à son propos sont radicalement différentes de celles relatives à l'histocompatibilité tissulaire. Le danger ne réside plus en effet dans l'instrumentalisation du corps d'autrui mais dans l'utilisation des données génétiques propres du patient. Le questionnement éthique est donc nouveau. Il est relatif à l'émergence dans la relation médicale de ces données abstraites, figées, aux côtés de l'individu concret, en perpétuelle évolution ; à l'apparition du corps numérique ${ }^{7}$ du patient, d'un corps abstrait composé de données codées ou chiffrées ${ }^{8}$, dans l'ombre de son corps réel'. Il s'inscrit dans cette évolution en vertu de laquelle le naturel autrefois opposé au surnaturel en ce qu'il apparaissait visible, audible, tangible est devenu à la suite des progrès scientifiques "un carrefour d'abstractions" ${ }^{10}$ dont la toile est l'exemple topique. Le corps numérique n'est ainsi qu'une nouvelle composante de la réalité numérique dont les mots clés, information, transparence, secret, preuve, fichier, illustrent que l'enjeu du débat est l'information, autour de laquelle "la transparence et le secret se livrent un débat sans relâche"".

Il est donc douteux que le chemin se révèle aisé pour appréhender cette nouvelle réalité qu'est la projection de l'individu dans une autre identité abstraite, génétique, qui lui est propre que nous proposons d'appeler son corps numérique, d'autant plus que cette avancée médicale est lente et diffuse. Il n'y a pas un évènement tel que la naissance de la brebis Dolly pour inciter le législateur à intervenir. Il serait en revanche pertinent que la prochaine révision des lois bioéthiques aborde les questionnements relatifs à ce corps numérique devenu réalité.

C'est l'apparition de la médecine personnalisée qui signe l'émergence du corps numérique dans la réalité médicale. Le concept de médecine personnalisée

\footnotetext{
${ }^{7}$ L'expression est celle du Pr. Hervé ChneIweiss, cité in Rapport, p. 155.

${ }^{8}$ Le Petit Robert, V. Numérique : "qui est représenté par un nombre".

${ }^{9}$ Nous ne nous attacherons pas au génome humain dépassant la personne individuelle en ce que chaque être humain porte une part du patrimoine génétique de l'humanité et qui permet de rattacher la protection de l'intégrité de l'espèce humaine à la dignité de la personne comme l'a fait le Conseil constitutionnel en 1994, V. sur ce point, F. Bellivier, Le patrimoine génétique humain : étude juridique, Thèse droit privé, Paris I, 1997 ; B. Mathieu, Génome humain et droits fondamentaux, Economica-PUAM, coll. Droit public positif, 1999 ; X. BıoY, La dignité : questions de principes, in Justice, éthique et dignité, Presses universitaires de Limoges, 2006, p. 69-70.

${ }^{10}$ Catala, Le droit à l'épreuve du numérique, Jus ex machina, PUF, 1998, épilogue, p. 343

${ }^{11}$ Ibid.
} 
participe en effet grandement à désincarner la personne au moyen du codage de ses particularités génétiques puisqu'elle est techniquement fondée sur deux outils majeurs pour observer l'ADN qui ont connu des progrès fulgurants et qui peuvent être aujourd'hui utilisés à un coût de plus en plus raisonnable, le séquençage à haut débit du génome génome ${ }^{12}$ et le génotypage ${ }^{13}$.

Concept encore flou, la médecine personnalisée connaît des définitions plus ou moins larges qui englobent ou non la prise en compte de l'environnement, le mode de vie d'un individu qui se réduisent ou non à la pharmacogénétique ${ }^{14}$. Mais elles s'accordent toutes pour reconnaître que cette nouvelle médecine s'appuie sur les caractéristiques génétiques de l'individu ${ }^{15}$. C'est ainsi que dans son acception la plus stricte, il s'agit de la personnalisation des traitements à partir de l'information génétique du patient, l'information génétique regroupant tant les informations génétiques structurelles du patient que les informations génétiques somatiques (analyse génomique d'une tumeur par exemple). Dans une acception plus large, la médecine personnalisée est dite celle des 4P : personnalisation autour du traitement d'une maladie ; préventive, la dimension de prévention étant importante ; prédictive car le patient peut recourir à des tests ; participative au sens où l'individu deviendrait l'acteur de sa propre santé.

La médecine personnalisée n'est donc pas en réalité une médecine de rapprochement avec le patient, de partage de son intimité, comme son nom le suggère, mais une médecine de précision en ce qu'elle s'appuie sur les données individualisées du patient découvertes grâce aux formidables progrès de la science. Elle met fin en ce sens à la médecine de masse dont les médicaments dits blockbusters

${ }^{12}$ Le séquençage détermine l'ordre d'enchaînement des nucléotides pour un fragment d'ADN donné.

${ }^{13}$ Le génotypage vise à déterminer la nature d'une variation génétique à une position spécifique dans le génome d'un individu.

${ }^{14}$ L'European Science Foundation en formule par exemple une définition très large : pratique médicale dont l'individu est le centre, à la fois précise et adaptée à ses caractéristiques biologiques qui englobent ses données génétiques, ses taux de protéine, ses biomarqueurs et prend en compte son environnement, son mode de vie, ses habitudes alimentaires, Personnalized Medicine for the European Citizen towards more precise medicine for the diagnosis, traitment and prevention of disease, october 2012.

15 "Sans nier que d'autres caractéristiques puissent venir alimenter la personnalisation du traitement, la Commission considère toutefois que l'utilisation de l'information génétique du patient constitue le cœur de ce paradigme", "Soins de santé personnalisés", Commission de l'éthique en sciences et en technologie, 2012, Québec, p. 9. 
soignent une pathologie, pour devenir une médecine presque sur mesure dont le médicament dit stratifié est adapté dans son indication et dans son dosage au métabolisme du patient.

La médecine personnalisée a été développée à l'origine par le laboratoire pharmaceutique Roche. Elle est fondée sur le fait qu'un même médicament peut provoquer des réactions différentes selon les patients. Ce laboratoire a ainsi élaboré le premier médicament dit stratifié, l'Herceptine, pour traiter le cancer du sein en ce qu'il est possible d'anticiper les effets du traitement positifs ou nuls grâce à un test génétique sur les patients. Ce médicament n'agit en effet que sur les femmes qui surexpriment le gène HER-2. Il est donc commercialisé avec un kit de diagnostic HER-2. L'impact de cette nouvelle approche est très important : efficacité accrue, effets secondaires réduits, absence de temps perdu et de ressources gaspillées dans un traitement inopérant. L'objectif de la médecine personnalisée est en effet non seulement de "prescrire les bons médicaments aux bons malades mais aussi d'éviter les traitements inutiles, toxiques qui sont aujourd'hui largement prescrits" ${ }^{\prime 16}$. D'autres médicaments ont depuis lors rejoint l'Herceptine, l'Abacavir dans le traitement du Sida, l'Interféron dans le traitement de l'hépatite $C$, mais surtout des anticancéreux ${ }^{17}$. Le test génétique dit test compagnon permet de déterminer l'efficacité d'un médicament ou sa posologie afin de garantir une adaptation maximale du traitement aux particularités génétiques du patient. Il commence à s'imposer dans la médecine contemporaine : il est déjà indiqué dans le résumé des caractéristiques d'un certain nombre de médicaments ${ }^{18}$ la nécessité de réaliser cet examen. Le nombre de tests génétiques a ainsi considérablement augmenté entre 2009 et $2012^{19}$.

\footnotetext{
16 Th. Tursz, Stratégies innovantes en cancérologies, implications sanitaires et économiques, séance commune des Académie des sciences et Académie Nationale de médecine, 23 avril 2013.

${ }^{17}$ On peut ainsi citer l'Imatinib ou Glivec dans le traitement de la leucémie myéloïde chronique. V. F. GuiLнот, La leucémie myéloïde chronique : traitement ciblé par inhibiteurs de tyrosine kinase, Séance commune,

${ }^{18} \mathrm{Au} 1^{\mathrm{er}}$ octobre 2013, on pouvait estimer leur nombre à 17.

${ }^{19}$ On compte en 2012 en France, 82756 tests de cytogénétiques et 416767 tests de génétique moléculaire qui couvrent aujourd'hui plus de 1500 maladies. En 2009, ces tests génétiques moléculaires n'étaient que de 271330 et ne couvraient que 1000 maladies, Chiffres rapportés par Mme Prada-Bordenave, Directrice générale de l'Agence de la Biomédecine, OPECST, Rapport précité, p. 110.
} 
L'oncologie est pour l'instant le domaine essentiel de la médecine personnalisée $^{20}$ à tel point que, dans les essais actuellement en cours, notamment l'essai SHIVA à l'Institut Curie, c'est la carte génétique de la tumeur et non sa localisation qui décide du traitement du cancer ${ }^{21}$. C'est un changement de paradigme médical identique à celui que l'on a connu lors de la découverte des antibiotiques : le traitement est spécifique du germe et non de l'organe ${ }^{22}$. Mais des traitements ciblés sont annoncés dans le domaine des neuropathologies (maladie d'Alzheimer), l'asthme, le diabète ou les maladies cardiovasculaires. C'est ainsi que le rapport de la commission "Innovation 2030" prévoit une généralisation de cette médecine : "l'individu et ses caractéristiques propres seront plus que jamais au cour de la médecine de demain" "23. L'affirmation de ce corps numérique ouvre même la porte au projet de Carte d'Identité Métabolique (CIME) ${ }^{24}$, à la création d'un avatar personnalisé de cancer voire d'un double biologique

Les dispositions applicables au corps numérique s'avèrent, au regard de cette évolution, largement insuffisantes et impropres à prendre en considération les nouveaux enjeux qui se profilent. Elles se retrouvent principalement au sein du chapitre III du Code civil consacré à "l'examen des caractéristiques génétiques d'une personne et (de) l'identification d'une personne par ses empreintes génétique".

La protection de l'examen des caractéristiques génétiques de la personne n'est pas spécifique en ce qu'elle est quasiment semblable à celle de l'imagerie cérébrale qui relève du chapitre suivant, le chapitre IV, "De l'utilisation des techniques d'imagerie cérébrale". Les articles 16-10 et 16-14 sont ainsi identiques sur le fond imposant d'une part la finalité médicale ou scientifique de l'examen et d'autre part le principe du consentement préalable par écrit à sa réalisation. L'article 16-13 seul souligne l'aspect sensible de cette information en précisant que "nul ne peut faire l'objet de discriminations en raison de ses caractéristiques génétiques".

${ }^{20}$ Voir OPECST, Rapport précité, p. 41. V. également La médecine personnalisée en cancérologie, séance commune des Académie des sciences et Académie Nationale de médecine, 23 avril 2013 ; A. Placi, B. Bleton, D. Haggiag Mazeau, Aude d'Ussel, dir. A. Paci, Médecine personnalisée et cancer, Organiser et financer l'accès à l'innovation, Presses de l'Institut Gustave Roussy, coll. Santé/Recherche, 2013,

${ }^{21}$ V. OPECST, Rapport précité, p. 48-49.

${ }^{22}$ Pr. Laurebt Deogs, cité in OPECST, Rapport précité, p. 53.

${ }^{23}$ Cité in OPECST, Rapport, p. 17.

${ }^{24}$ V. J-M. Grognet, OPECST, Rapport précité, p. 34. 
L'encadrement proposé est de plus commun avec celui de l'empreinte génétique alors même que les enjeux sont différents. La réalisation de l'empreinte génétique est fondée uniquement sur le séquençage de l'ADN non codant qui détermine l'unicité de chacun. Elle informe seulement sur la structure du matériel génétique mais ne révèle pas l'activité cellulaire du matériel génétique qui renseigne sur les caractéristiques génétiques mêmes de la personne. Elle ne peut donc servir qu'une finalité, l'identification de la personne parmi d'autres, sans dévoiler son identité génétique propre. Sa fonction policière est évidente et l'article 16-11 du Code civil a significativement été complété par une loi de 2011 portant sur la sécurité intérieure. Les nouvelles données biométriques qui prennent en compte l'iris, la voix, les veines, la forme des mains servent également la même finalité d'identification de la personne même si le risque de "traçage" des individus se profile et suscite également des questionnements ${ }^{25}$.

L'examen des caractéristiques génétiques propre donne en revanche à voir la spécificité génétique de la personne même à travers l'examen de l'activité cellulaire de son matériel génétique. Il y a une différence fondamentale entre les résultats : le premier est simplement d'affirmer que A n'est pas B alors que l'autre dévoile qui est génétiquement $\mathrm{A}$.

En tant que données de santé, les données génétiques sont également protégées par la 6 janvier 1978 modifiée ${ }^{26}$. La réforme de cette loi par celle du 6 août 2004, transposant la directive européenne 95/46/CE du 24 octobre 1995, intègre l'ensemble des données de santé ${ }^{27}$ dans la catégorie des données dites "sensibles" et, le traitement des données de santé, qu'il soit mis en oeuvre par une entité

${ }^{25} \mathrm{C}$. BYK, Biométrie et Constitution, est-il déjà trop tard pour les libertés publiques ?, JCP 2008, I, 154 ; D. Siroux, La "biométrisation" de l'identité, de la sécurité à la surveillance, GP, 2007, 3652 .

${ }^{26} \mathrm{La}$ donnée personnelle est "toute information relative à une personne physique identifiée ou qui peut être identifiée, directement ou indirectement par référence à un numéro d'identification ou à un ou plusieurs éléments qui lui sont propres", Art. 2 de la loi du 6 janvier 1978 modifiée par la loi n 2004-801 du 6 août 2004.

${ }^{27} \mathrm{Il}$ est à noter qu'au niveau européen, un projet de règlement sur la protection des données prévoit de mentionner pour la première fois au nombre des données sensibles, les données génétiques. La Commission des libertés civiles, de la justice et des affaires intérieures (LIBE) du Parlement européen a adopté le 21 octobre 2013 la proposition de règlement de l'Union européenne sur la protection des données personnelles. Cette proposition s'attache surtout à assurer la sécurité des données génétiques : il faut veiller à ce que les données soient conservées et traitées par le responsable du traitement dans des conditions de nature à garantir leur confidentialité mais aussi leur intégrité. 
publique ou privé, est ainsi étroitement encadré et soumis au respect de règles contraignantes, relevant notamment du régime de l'autorisation par la CNIL. Mais cette loi présente également des insuffisances, notamment dans son champ d'application. La protection de la CNIL vise principalement un ensemble de données collectées en vue de leur traitement informatique ${ }^{28}$. Ce sont ces mêmes données rassemblées à l'occasion de "collections d'échantillons biologiques humains" dites biothèques qui font également l'objet d'une protection spécifique toujours par le biais de la $\mathrm{CNIL}^{29}$. Mais surtout la protection offerte par la loi de 1978 ne prévoit qu'un simple droit d'opposition de la personne qui paraît insuffisant.

A l'issue de cette analyse, il apparaît que les données génétiques de la personne sont mal protégées. C'est compréhensible pour une donnée de santé ponctuelle qui vise justement à renseigner sur l'état de santé d'un individu à l'instant " $t$ ", qui perdra en principe toute pertinence à l'instant " $t+1$ " et qui ne peut pas être exclusivement attachée à une personne dans la mesure où cette donnée peut être la même que celle obtenue à partir d'un autre individu. Mais un tel manque de protection est préoccupant lorsque la donnée de santé renseigne sur l'identité génétique même de la personne, donnée relativement figée qui dévoile la structure même de la personne.

Enfin, les textes actuels plus spéciaux sont très marqués par la médecine prédictive, la première concernée par la génétique, qui a pour finalité le diagnostic d'une maladie génétique. Or la médecine prédictive a un fort retentissement familial et la plupart des textes ont justement pour objet d'encadrer la transmission d'un tel diagnostic à la parentèle du patient et ne se préoccupent pas de l'intérêt de transmettre ces données au patient lui-même dans la mesure où elles ont un intérêt médical évident. Or les données issues de la médecine personna-

${ }^{28}$ La loi s'applique en effet prioritairement au traitement automatisé de données, ce qui suppose qu'un certain nombre de données soient réunies. "Constitue un traitement de données à caractère personnel toute opération ou tout ensemble d'opérations portant sur de telles données quel que soit le procédé utilisé et notamment la collecte, l'enregistrement, l'organisation, la conservation, l'adaptation ou la modification, l'extraction, la consultation, l'utilisation, la communication par transmission, diffusion, ou toute autre forme de mise à disposition, le rapprochement ou l'interconnexion, ainsi que le verrouillage, l'effacement ou la destruction" (L. n 78-17, 6 janv. 1978, art. 2). La loi s'applique également aux traitements non automatisés de données à caractère personnel dès lors qu'elles sont réunies en un fichier. Le législateur précise que constitue un fichier de données à caractère personnel tout ensemble structuré et stable de données à caractère personnel accessibles selon des critères déterminés (L. n 78-17, 6 janv. 1978, art. 2).

${ }^{29}$ Art. L 1131-4 et L. $1243-3$ et 4 du code de la santé publique. 
lisée vont bien au-delà du seul diagnostic d'une maladie génétique identifiée. Elles lui transmettent sa propre identité génétique, dont le contenu peut s'avérer anxiogène en ce qu'il renseigne sur des susceptibilités ou des prédispositions à plusieurs maladies s'inscrivant en dehors de tout protocole médical.

La question fondamentale que pose la médecine personnalisée est en conséquence celle du statut de ce corps numérique. Or, le statut du corps numérique qui désigne un ensemble de règles propres à en encadrer le régime invite immanquablement à s'interroger sur sa relation avec la personne humaine. Il apparaît que le corps numérique n'est pas la personne humaine et que cette affirmation conduit à une application nouvelle des principes protecteurs de la personne dans le cadre de la médecine personnalisée. Cependant ce corps numérique lui est tout de même puissamment lié et oblige à réfléchir à de nouveaux principes protecteurs de la personne.

\section{I - LE CORPS NUMÉRIQUE N'EST PAS LA PERSONNE : DE NOUVELLES APPLICATIONS DES PRINCIPES PROTECTEURS DE LA PERSONNE HUMAINE}

La personne humaine, l'homme dans son essence, implique le corps. C'est ainsi que corps humain, "substratum de la personne" ${ }^{\text {"30 }}$ se confond avec celle-ci. Le principe d'inviolabilité du corps énoncé à l'article 16-1 alinéa 2 exprimant le fameux noli me tangere (ne me touchez pas) "trouve son fondement dans l'idée que le corps humain, incarnation de la personne, participe même de l'essence de l'homme et doit bénéficier du respect dû à celui-ci'”31.

Cette identification entre le corps numérique et la personne n'existe pas. Le corps numérique ne peut être touché, palpé, tranché. Il est abstrait, sans consistance réelle, incorporel, puisque seulement composé de données. Il n'est pas de l'essence de la personne. Une balle tirée dans un une série de signes abstraits n'a aucune chance de tuer la personne.

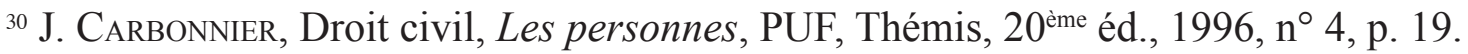

${ }^{31}$ Assemblée Nationale, Exposé des motifs du projet de loi relatif au corps humain, $\mathrm{n}^{\circ} 2599,25$ mars 1992, p. 3.
} 
Le corps numérique n'est d'ailleurs pas forcément révélé au cours d'une existence humaine ${ }^{32}$, comme ce fut le cas pendant des millions et des millions d'années et comme c'est toujours le cas pour une très grande majorité d'entre nous. On peut vivre sans corps numérique contrairement au corps appelé fort à propos corps humain en ce qu'il porte la vie humaine ${ }^{33}$.

Il est donc impossible de rattacher le corps numérique à la personne et de lui appliquer les principes dégagés à son égard.

En revanche, les principes dégagés à propos de la personne humaine permettent de dessiner la place du corps numérique. En assimilant la personne et son corps, les lois dites bioéthiques ont posé deux principes fondamentaux formulés à l'article 16 du Code civil, la primauté de la personne humaine et sa dignité, qui trouvent de nouvelles applications dans le cadre de la médecine personnalisée.

\section{A - L'application du principe de la dignité de personne humaine : le corps numérique n'efface pas la personne}

La dignité de la personne humaine déclarée valeur essentielle de l'humanité, et sa sauvegarde qui a été constitutionnalisée dans notre pays à l'occasion justement du contrôle exercé par le Conseil constitutionnel sur les lois dites bioéthiques du 29 juillet $1994^{34}$, peut trouver une nouvelle application grâce au progrès scientifique qu'est la médecine personnalisée en ce qu'il permet de neutraliser le risque de dépersonnalisation et donc de dégradation de la personne humain qu'elle transporte ${ }^{35}$. C'est la conséquence effroyable de toute avancée scientifique lorsqu'elle est employée à mauvais escient comme le suggère le Conseil constitutionnel lorsqu'il invoque à l'appui de sa décision la première phrase du premier alinéa de la Constitution de 1946. Le peuple français y proclame en effet les droits inaliénables et sacrés de tout être humain sans distinction de race, de

\footnotetext{
${ }^{32}$ Le droit des brevets vacille d'ailleurs, tellement le génome, préexistant, a des difficultés à être appréhendé comme une "invention" de l'homme, V. N. Belrhomari, Génome humain espèce humaine et droit, préf. G. TeBOuL, L'Harmattan, 2013, spéc. p. 83 et s.

${ }^{33} \mathrm{Il}$ a d'ailleurs un statut différent dès lors que la vie humaine s'est éteinte et que le corps humain devient cadavre.

${ }^{34}$ Plus exactement, il a été jugé que "la sauvegarde de la dignité humaine contre toute forme d'asservissement et de dégradation est un principe à valeur constitutionnelle", C. Const. 27 juillet 1994, DC n 94-343-344.

${ }^{35}$ Ce risque de dépersonnalisation est souligné par tous les spécialistes, V. OPECST, Rapport, spéc. p. 63 et s.
} 
religion, ni de croyance, et y exprime l'aversion suscitée à l'égard de la science par les expériences hitlériennes.

Mais au sein de la médecine personnalisée, la dégradation de l'homme est inhérente à l'exercice même de l'art médical et ne réside pas dans une de ses applications dévoyées. La personne est inévitablement traduite en données abstraites et le risque de dépersonnalisation participant immanquablement à sa dégradation est immédiat. Le principe de dignité servirait de rempart à cette dépersonnalisation spontanée de la personne puisqu'il rappelle que la personne ne saurait être réduite à une masse de données, aussi précises et individualisées soient-elles. La personne ne saurait être un point dans un nuage de points selon l'expression de $\mathrm{J}-\mathrm{Cl}$ Ameisen $^{36}$. Le respect de sa dignité impose de la considérer d'abord comme une personne humaine, incarnée, avec toute son épaisseur psychologique, son histoire, ses espoirs, ses peurs, ses joies, ses faiblesses, ses forces, bref impose de l'appréhender dans son incommensurabilité ${ }^{37}$.

La Déclaration universelle sur le génome humain et les droits de l'homme proclamée le 11 novembre 1997 a déjà posé un principe en ce sens : "chaque individu a droit au respect de sa dignité (...) cette dignité impose de ne pas réduire les individus à leurs caractéristiques génétiques et de respecter leur caractère unique et leur diversité". C'est la même idée qui sous-tend l'arrêté du 27 mai 2013 définissant les règles de bonnes pratiques applicables à l'examen des caractéristiques génétiques d'une personne à des fins médicales lorsqu'il dispose moins solennellement et plus spécialement que "l'individu doit rester au centre des préoccupations des acteurs du diagnostic des maladies génétiques" ${ }^{38}$.

Le principe de dignité de la personne humaine, "principe à vertu fécondante"39, pourrait trouver là des déclinaisons nouvelles, révélant notamment des droits attachés à la personne même du patient qui jusqu'à présent n'avaient donné lieu à aucun contentieux. Il permettrait par exemple d'imposer un colloque singulier

${ }^{36}$ Cité in OPECST, Rapport, p. 63.

${ }^{37} \mathrm{~V}$. en ce sens l'article L. 4127-35 du code de la santé publique issu du code de déontologie médicale : "Le médecin doit à la personne qu'il examine, qu'il soigne ou qu'il conseille une information loyale, claire et appropriée sur son état, les investigations et les soins qu'il lui propose. Tout au long de la maladie, il tient compte de la personnalité du patient dans ses explications et veille à leur compréhension".

${ }^{38}$ Arrêté du 27 mai 2013 définissant les règles de bonnes pratiques applicables à l'examen des caractéristiques génétiques d'une personne à des fins médicales, $J O$ du 7 juin 2013.

${ }^{39} \mathrm{M}$. Gobert, in La dignité de la personne humaine, dir. M-L PAVIA, T. REVET, Economica, 1999, Rapport de synthèse, p. 163. 
entre patient et médecin, alors que le test génétique permettrait seul de décider du traitement adéquat. Il imposerait également, justement dans le cadre de ce colloque singulier, de ne pas se focaliser sur les seules données abstraites mais de prendre en considération le mode de vie du patient, son environnement, ses comportements, son alimentation pour adapter au mieux le traitement à sa personne, comme le postule déjà les définitions les plus larges de la médecine personnalisée.

Il est aussi susceptible de consolider le droit au refus de soins. Celui-ci s'inscrit pour l'instant dans un contexte traditionnel à savoir que le traitement proposé au patient est celui qui a démontré une efficacité maximale eu égard à sa pathologie. S'il le refuse pur des raisons qui lui sont propres, le médecin tente alors de lui proposer une alternative a priori moins efficace. Ce cas de figure est d'ailleurs relativement rare, le refus de soin s'inscrivant généralement dans un désir de fin de vie. Dans le cadre de la médecine personnalisée, le patient peut bénéficier d'un médicament ayant démontré une efficacité maximale seulement si les analyses génétiques résultant du test compagnon correspondent à son indication. Si cette condition n'est pas satisfaite, il se voit alors proposer un traitement plus classique, comme une chimiothérapie dans le cadre d'un cancer. La médecine personnalisée participe en ce sens à creuser les inégalités entre les patients atteints de cancer ${ }^{40}$. Mais le patient peut exprimer le choix de refuser ce traitement et de recourir à ce traitement innovant. Le droit d'accéder à ce traitement fondé notamment sur le respect de sa dignité lui semble difficilement contestable, dans la mesure où ce traitement n'est pas nocif pour lui. Il faudrait cependant qu'il en assume le coût généralement élevé ${ }^{41}$ puisque ce traitement n'est en principe remboursé par la Sécurité sociale que s'il a démontré son efficacité c'est-à-dire si le test compagnon est positif ${ }^{42}$. Ce serait alors un privilège réservé aux plus riches. A une inégalité génétique se substitue en quelque sorte une inégalité sociale. La médecine personnalisée semble constitue par essence un facteur d'inégalité entre les patients.

Aux cotés du principe de dignité, l'autre principe dégagé par les lois bioéthique est celui de la primauté de la personne. Ce principe fondamental n'a pour

${ }^{40}$ Les inégalités socio-professionnelles ont déjà un impact fort sur le diagnostic et le traitement du cancer.

${ }^{41}$ Le traitement d'un patient au Zelboraf coûte par exemple 9800 euros par mois.

${ }^{42}$ Sa prise en charge en France est déjà un atout considérable. Certains pays comme l'Angleterre ou l'Allemagne rejettent le remboursement de médicaments issus de la médecine personnalisé lorsqu'ils sont trop chers. 
l'instant que rarement été invoqué mais il permet dans le cadre de la médecine personnalité d'en déduire un principe très important : la personne primant, le corps numérique doit être au service de la personne et ne saurait lui nuire.

\section{B- L'application du principe de primauté de la personne : le corps numérique est au service de la personne}

Le principe précis qui peut en être déduit est que le corps numérique, les données génétiques qu'il contient, ne sauraient être utilisées contre la personne. Comme on ne peut subroger contre $\operatorname{soi}^{43}$, on ne pourrait non plus révéler son corps numérique contre soi.

L'enjeu est important car le corps numérique constitue une masse de données considérables qui sont susceptibles d'être utilisées contre la personne notamment pour contrôler ses comportements et susciter des devoirs envers elle-même. Le potentiel de la médecine personnalisée et notamment la pharmacogénomique ne pourra en effet se réaliser que si des chercheurs peuvent avoir accès à l'information génétique de nombreux individus dans des essais cliniques de grande envergure. L'accès en masse à ces tests génétiques pose la question de son impact dans la mesure où ces tests ont une valeur prédictive croissante sur des maladies de plus en plus nombreuses et renforce incontestablement la médecine prédictive.

Le concept de médecine prédictive qualifie la médecine ayant pour but de détecter une prédisposition biologique à une maladie afin de retarder, d'atténuer voire d'empêcher son apparition. Cette médecine préventive personnalisée repose sur l'identification de variations génétiques qui signalent une prédisposition ${ }^{44}$ ou même seulement une susceptibilité individuelle à déclencher une maladie dans un environnement donné. Pour être légitimes ces tests doivent révéler un risque supérieur aux influences environnementales et permettre une action préventive ou curative comme c'est le cas pour les gènes de prédisposition aux cancers du

${ }^{43}$ L'article 1252 du code civil dispose que la subrogation "ne peut nuire au créancier lorsqu'il n'a été payé qu'en partie; en ce cas il peut exercer ses droits, pour ce qui lui reste dû, par préférence à celui dont il n'a reçu qu'un paiement partiel". Il met en œuvre l'adage "nemo contra se subrogasse censetur" qui exprime notamment l'idée que le créancier originaire subrogeant payé en partie seulement n'a pas la volonté de subroger à son encontre mais bien de se réserver la meilleure position par rapport au créancier subrogé.

${ }^{44}$ La prédisposition génétique d'une personne est un risque endogène, d'origine génétique, de contracter une maladie ou de la transmettre à ses enfants, risque dont la réalisation incertaine est soumis à des facteurs catalyseurs extérieurs, A. Marais, La prédisposition génétique, Thèse Paris II, dir. M. GOBERT, 2000, nº 25, p. 11. 
sein familiaux (BRCA1 et BRCA2). Mais, même dans cette hypothèse, l'anomalie génétique n'est bien souvent ni nécessaire ni suffisante pour avoir la maladie. L'utilité médicale de ces tests n'est alors pas établie. Cependant, quel que soit le cas de figure, le patient est incontestablement responsabilisé dans la mesure où il a la possibilité d'adapter son mode de vie à sa spécificité génétique. Par exemple une personne dont les données génétiques révèlent une prédisposition au diabète est incitée à modifier ses habitudes alimentaires, une personne prédisposée aux $\mathrm{AVC}$ à vivre dans un environnement calme. La personne devient alors actrice de sa propre santé à tel point que l'apparition de la maladie à laquelle la personne se savait prédisposée pourrait être considérée comme une faute qui lui serait imputable.

La question est celle de la sanction de la défaillance dans l'adaptation de son mode de vie dans l'éventualité où la maladie s'est déclenchée. Elle devrait en vertu du principe de primauté de la personne se cantonner à la sanction naturelle à savoir l'altération de son état de santé sans que le droit n'en prévoie sous peine de transformer ce conseil médical en devoir juridique et aboutir ainsi à un véritable contrôle des comportements. Ce serait le cas si le non respect de cette alimentation démontré au moyen de prises de sang régulières conduisait à une absence de prise en charge de son diabète par la Sécurité sociale.

Cette projection n'est pas irréaliste puisqu'un arrêté du 22 octobre $2013^{45}$ est venu conditionner le remboursement du port d'un masque de traitement du syndrome de l'apnée du sommeil à la durée et à la régularité du port du masque par le patient dont le contrôle est réalisé par télésurveillance. Le patient qualifié d' "inobservant" c'est-à-dire n'ayant pas rempli les conditions d'utilisation définies par l'arrêté en cause, voit son droit à remboursement progressivement réduit puis supprimé. Le Conseil d'Etat a cependant suspendu l'application de cet arrêt en référé arguant d'un doute sérieux sur sa légalitét ${ }^{46}$.

La liberté de l'individu, protégée notamment par l'article 4 de la déclaration de droits de l'homme et du citoyen, s'en trouverait en effet fortement entravée. Le respect de sa vie privée, garanti par l'article 8 de la Convention européenne des droits de l'homme, serait également profondément ébranlé en ce que ce contrôle de leur comportement constitue une intrusion excessive dans leur vie intime. Ces atteintes seraient d'autant plus graves que les prédispositions ne peuvent dans

\footnotetext{
${ }^{45}$ Arrêté du 22 octobre 2013 modifiant les modalités de prise en charge par l'assurance maladie du traitement de l'apnée du sommeil.

${ }^{46} \mathrm{CE}$, ord. du 14 février 2014, n 374699. Le Conseil d'Etat met en cause la compétence d'un simple arrêté pour prendre de telles dispositions.
} 
l'avenir que s'ajouter les unes aux autres au fil des progrès scientifiques fulgurants en la matière. On peut ainsi imaginer une personne interdite de manger du sucre (prédisposition de diabète), de consommer de l'alcool (prédisposition à un cancer du foie), du tabac (prédisposition au cancer du poumon), de vivre dans un environnement stressant tel qu'une grande ville (prédisposition à un AVC), ou dans l'obligation de stimuler régulièrement sa mémoire à l'aide d'un professionnel (prédisposition à la maladie d'Alzeihmer). On pourrait y voir le spectre d'une "société hygiéniste" ${ }^{47}$. La primauté de la personne induit en ce sens le principe selon lequel les données génétiques de la personne ne sauraient être utilisées contre elle. Il paraît préférable de substituer à une telle médico-surveillance une politique d'éducation à la santé.

Plus généralement, ces données, dans le cadre de ce principe, ne pourraient être utilisées par des tiers à l'encontre de la personne, même si la personne a consenti à leur diffusion. L'instauration de ce principe s'inscrirait dans la logique de neutralisation de ces données déjà initiée par des textes spéciaux à l'égard des assurances invalidité et décès ${ }^{48}$ ou de l'accès à un emploi, un stage ou une formation $^{49}$. Il permettrait également de mettre en perspective certains textes qui manifestement ne répondent pas à ce principe et de s'interroger sur leur légitimité. C'est ainsi que l'article L. 1132-2 du code du travail prévoit une prise en compte des caractéristiques génétiques de la personne dans le monde du travail dès lors qu'elle "répond à une exigence professionnelle essentielle et déterminante (...) pour autant que l'objectif soit légitime et l'exigence proportionnée", ce qui ouvre la porte à des licenciements pour motifs de santé déguisés. Face à ce constat, le Comité Consultatif National d'Ethique a déjà critiqué ce texte pointant d'une part son imprécision mais surtout dénonçant sa substance même, affirmant que l'utilisation de ces données "ne devrait jamais avoir comme conséquence de réduire la prévention des risques professionnels en privilégiant l'élimination des salariés les plus exposés génétiquement plutôt que l'aménagement de l'environnement du travail" ${ }^{\text {,50 }}$. Il se prononce ainsi clairement pour que l'utilisation des tests génétique

\footnotetext{
${ }^{47}$ OPECST, Rapport précité, p. 146.

${ }^{48} \mathrm{CE}$, ord. du 14 février 2014, $\mathrm{n}^{\circ}$ 374699. Le Conseil d'Etat met en cause la compétence d'un simple arrêté pour prendre de telles dispositions.

${ }^{49}$ L'article L. 1132-1 du code du travail prévoit qu' "une personne ne peut être écartée d'une procédure de recrutement ou de l'accès à un stage ou à une période de formation en entreprise (...) en raison de (...) ses caractéristiques génétiques".

${ }^{50} \mathrm{CCNE}$, Avis $\mathrm{n}^{\circ} 46$, Génétique et médecine : de la prédiction à la prévention, 30 octobre 1995, p. 32.
} 
dans la mesure où elle est permise ne le soit que dans une perspective favorable au salarié, l'aménagement de son environnement de travail.

Ce principe permettrait plus généralement de protéger la personne à l'égard de tous les tiers susceptibles d'être intéressés par ces données. C'est l'ensemble des acteurs de l'assurance qui est évidemment en premier visé, pas seulement à l'égard des assurances invalidité/décès ${ }^{51}$, du monde du travail, mais également ceux du domaine sécuritaire. En effet, les politiques pénales ont toujours la tentation de détecter la prédisposition à une délinquance future, comme cela a déjà été démontré ${ }^{52}$.

Le corps numérique n'est certes pas la personne mais il entretient un lien puissant avec la personne. Il est en effet la projection même de la personne en données abstraites et il y a en ce sens autant de génomes que de personnes. Il ouvre la voie à la recherche de nouveaux principes protecteurs de la personne.

\section{II - LE CORPS NUMÉRIQUE EST PUISSAMMENT LIÉ À LA PERSONNE : LA RECHERCHE DE NOUVEAUX PRINCIPES PROTECTEURS DE LA PERSONNE}

Le corps numérique n'est pas le corps humain. Il n'en constitue pas plus un élément ou un produit. En revanche, ces données génétiques tant structurelles que somatiques constituent des données uniques pour chaque personne. Elles ne constituent pas des données ponctuelles relatives à l'analyse d'un élément ou d'un produit du corps humain comme l'imagerie cérébrale, susceptibles d'être en tout point semblables à celle d'une autre personne. Rassemblées au sein du corps numérique, elles constituent l'identité génétique de la personne. Il n'est ainsi pas neutre que soient utilisées les expressions "Carte d'Identité Métabolique" ${ }^{53}$ ou "signature génétique d'une tumeur" ${ }^{54}$.

\footnotetext{
${ }^{51}$ Comme cela a déjà été relevé, il est possible d'imaginer un assureur automobile refusant la formation d'un contrat avec une personne du fait d'une anomalie génétique, V. JESSICA EYNARD, Les données personnelles, quelle définition pour un régime de protection efficace?, Michalon, 2013, p. 124.

${ }^{52}$ Voir sur ce point, les développements de J. EynARD, op. cit. , p. 129 et s.

${ }^{53}$ Sur ce projet, V. J-M. GRoGNET, OPECST, Rapport précité, p. 34

54 "Actuellement on compte 28 plateformes de génétique moléculaire labellisées INCa réparties sur l'ensemble du territoire, permettant de fournir la signature génétique de la tumeur de chaque patiente, OPECST, Rapport précité, p. 43.
} 
Cette identité génétique doit fondamentalement être reliée à la personne. C'est ainsi qu'il paraît opportun d'affirmer le caractère "personnellissime" de ces données et leur assurer une protection maximale au regard des tiers en dégageant le principe que chacun a droit à la protection de son identité génétique. De même, les données génétiques ne doivent pas être considérées isolément de la personne: il paraît essentiel d'apprécier l'intérêt de celle-ci à connaître de ses données. S'agissant de données médicales, elles doivent pouvoir être reliées à un pouvoir médical et s'inscrire en conséquence dans un protocole médical. Le savoir médical ne saurait avoir de valeur en soi détaché de ce pouvoir sauf à altérer la liberté de l'homme en lui transmettant des données anxiogènes.

\section{A-La protection de la personne contre elle-même : le cantonnement du dévoilement aux informations médicalement pertinentes}

La tentation est grande de tout connaître de son identité génétique. La donnée génétique renseigne sur la constitution de la personne même. Elle a donc un attrait considéré en elle-même en ce qu'elle satisfait une soif de connaissance à l'égard de soi-même ${ }^{55}$. Mais face à ce "déluge de données", semble pas qu'il soit, au regard des données acquises de la science, dans l'intérêt de la personne de connaître l'intégralité de ses données.

Les textes actuels sont très marqués par la médecine prédictive, la première concernée par la génétique, qui a pour finalité le diagnostic d'une maladie génétique. L'article R 1131-4 du code de la santé publique consacré aux conditions de prescription de cet examen prévoit ainsi que la personne est informée "des caractéristiques de la maladie recherchée, des moyens de la détecter, des possibilité de prévention et de traitement". De même l'article R 1131-5 distingue deux situations qui n'ont aucun intérêt à l'être dans le cadre de la médecine personnalisée, celle d'un patient présentant le symptôme d'une maladie génétique et celle d'une personne asymptomatique mais présentant des antécédents familiaux. Cette médecine prédictive a en effet un fort retentissement familial et la plupart des textes ont justement pour objet d'encadrer la transmission d'un tel diagnostic à la parentèle du patient et ne se préoccupent de l'intérêt de transmettre ces données au patient lui-même dans la mesure où elles ont un intérêt médical évident.

\footnotetext{
${ }^{55}$ La donnée génétique se distingue là encore radicalement de la donnée de santé traditionnelle qui n'a d'intérêt que parce qu'elle s'inscrit dans un protocole médical.

${ }^{56}$ M. Ashween Peerbay, OPECST, Rapport précité, p. 133.
} 
Ces textes sont donc insuffisants au regard de cette nouvelle médecine. L'information imposée préalablement au test compagnon n'est pas appropriée, ayant été réfléchie au regard de la première finalité du test génétique, la plus ancienne, le diagnostic d'une maladie génétique. Il serait important d'enrichir cette obligation d'information pour l'adapter à la médecine personnalisée. Le patient doit par exemple être informé précisément de la finalité du test, à savoir le choix du meilleur traitement, et des conséquences du résultat de ce test afin qu'il puisse bien comprendre que le résultat négatif du test n'est pas exclusif d'un traitement.

Mais il convient surtout de s'interroger sur l'objet du consentement du patient obtenu préalablement au test génétique. L'objet de son consentement vise incontestablement à autoriser l'atteinte corporelle mineure que constitue le prélèvement qui sinon aurait été illicite en vertu du principe d'inviolabilité de la personne. Mais le consentement est en réalité multiple d'abord en ce que le patient consent aussi à l'analyse de ces informations génétiques mais surtout à la transmission de ces informations. La question qui se pose est de savoir quelles informations doivent lui être transmises en vertu de ce consentement. Que faire des résultats du test qui transmettent plus d'informations que celles exclusivement nécessaires au traitement, les informations dites secondaires ${ }^{57}$ ? Que faire par exemple si le test compagnon, au-delà de son résultat positif ou négatif eu égard au traitement, révèle une prédisposition au cancer du sein ou une susceptibilité au diabète ? C'est une question importante qui a été soulevée notamment par l'Académie des sciences et l'Académie nationale de médecine en avril 2013 réunies lors d'une séance commune sur le thème de la médecine personnalisée $e^{58}$.

En l'absence de texte précis, le médecin est aujourd'hui seul juge des informations à transmettre au patient, renouant avec le paternalisme médical en vertu duquel le médecin avait le droit de décider de la pertinence des informations à transmettre au malade. Pourtant le secret médical, puisque c'est bien de cela qu'il s'agit, a depuis lors subi une métamorphose considérable. Il n'est plus entre les mains du médecin mais est devenu un droit des patients. Et le patient a acquis le droit à une information complète et exhaustive ${ }^{59}$. Le droit au mensonge dans

\footnotetext{
${ }^{57}$ Nous empruntons cette expression à la Commission de l'éthique en sciences et en technologie du Québec, Rapport précité, 2012, p. 19

${ }^{58}$ Question 3, séance commune des Académie des sciences et Académie Nationale de médecine, 23 avril 2013.

${ }^{59}$ L'article L. 1111-2 du code de la santé publique précise aujourd'hui que l'information doit porter sur "les risques fréquents et graves".
} 
l'intérêt du malade a ainsi disparu du code de déontologie au profit du droit de la personne à être tenue dans l'ignorance d'un diagnostic ou d'un pronostic ${ }^{60}$. Le savoir médical est donc désormais tout entier au pouvoir des patients. Dans le cadre de la médecine personnalisée, il paraît donc urgent de légiférer sur ce point et d'opter entre deux alternatives.

Il est possible considérer dans la logique des principes précédemment dégagés que le patient est seul maître des informations contenues dans ses gènes et qu'il doit exprimer son choix lors du consentement au test compagnon quant à sa volonté de connaître l'intégralité des informations transmises ou de se cantonner à celles médicalement pertinentes. Dans la mesure où il doit exprimer un tel choix, son consentement doit être éclairé quant à la nature des informations susceptibles de lui être transmises, à leur impact psychologique, notion bien connue déjà en génétique, mais également dans le domaine des prélèvements $\mathrm{d}^{\prime}$ organes ${ }^{61}$. Les informations transmises porteront en effet principalement sur des éléments anxiogènes comme un diagnostic de prédisposition voire de susceptibilité auxquels la médecine ne pourra généralement apporter aucune réponse thérapeutique ou préventive. Le savoir du patient se déconnecterait alors du pouvoir médical mais également de sa démarche initiale, à savoir le choix d'un traitement d'une maladie existante.

Il paraît probablement plus raisonnable de choisir la seconde alternative qui est de poser un principe en vertu duquel seules les informations médicalement pertinentes notamment au regard de la finalité médicale du test seront transmises au patient. Cette seconde alternative s'inscrirait dans la logique actuelle du droit

${ }^{60}$ Le décret du 7 mai 2012 a modifié l'article 4127-35 du code de la santé publique y abrogeant la disposition suivante "toutefois, sous réserve des dispositions de l'article L. 1111-7, dans l'intérêt du malade et pour des raisons légitimes que le praticien apprécie en conscience, un malade peut être tenu dans l'ignorance d'un diagnostic ou d'un pronostic graves, sauf dans les cas où l'affection dont il est atteint expose les tiers à un risque de contamination". Il l'a remplacée par la disposition suivante actuellement en vigueur : 'lorsqu'une personne demande à être tenue dans l'ignorance d'un diagnostic ou d'un pronostic, sa volonté doit être respectée, sauf si des tiers sont exposés à un risque de contamination".

${ }^{61}$ L'article R. 1231-1 du code de la santé publique précise la teneur de l'information transmise au donneur éventuel : risques et conséquences éventuelles sur le plan physique et psychologique, ainsi que répercussions sur sa vie personnelle, familiale et professionnelle. Il est prévu une audition spéciale de la personne susceptible d'être prélevée afin que le comité d'expert compétent pour donner son autorisation au prélèvement puisse vérifier que l'information transmise a bien été comprise. 
français qui cantonne l'accès au test génétique ${ }^{62}$. Le savoir serait là directement lié au pouvoir médical et à la démarche du patient. L'information du patient au test serait dès lors plus aisée dès lors que les informations anxiogènes liées notamment à un diagnostic de prédisposition ou de susceptibilité ne seront pas transmises ${ }^{63}$. Mais c'est surtout le bien-être du patient qui est préservé puisqu'il est délivré d'une source d'angoisse qui peut être considérable. C'est également sa liberté de mener sa vie comme il l'entend, sa liberté personnelle, qui n'est pas entravée par des données médicales sans valeur.

Beaucoup de tiers sont également intéressés par ces nouvelles informations. Et l'affirmation d'un nouveau droit, celui de la protection de son identité génétique permettrait de parfaire ce régime juridique, en garantissant une protection de la personne contre les tiers.

\section{B - La protection de la personne contre les tiers : l'affirmation du droit à la protection de son identité génétique}

Il n'y a pas de disposition protectrice de l'identité génétique de la personne qui permettrait de rattacher les données génétiques à la personne même et de leur conférer ainsi une valeur supérieure aux autres données de santé. Il paraîtrait en ce sens opportun de ranger la protection de l'identité génétique dans les droits de la personnalité qui se définissent comme "des droits inhérents à la personne humaine, qui appartiennent de droit à toute personne physique pour la protection de ses intérêts primordiaux" ${ }^{\prime 64}$. Le droit de la personnalité qui permet "d'exiger d'autrui sa reconnaissance comme individualité distincte de toutes autre

${ }^{62}$ Les tests génétiques ne sont en principe accessibles que sur prescription médicale et ne peuvent être pratiqués que dans des laboratoires de biologie médicale autorisés à cet effet (C. santé publ, art. L. 1131-2-1). En conclusion d'un avis récent, le comité a annoncé que les problèmes éthiques posés par une éventuelle commercialisation de dispositifs médicaux de diagnostic in vitro permettant la réalisation de tests sans accompagnement médical, et notamment de tests génétiques feraient l'objet d'une réflexion globale, dans un prochain avis (Avis CCNE $n^{\circ} 119$, Les problèmes éthiques posés par la commercialisation d'autotests de dépistage de l'infection VIH, 21 février 2013).

${ }^{63}$ Sur le caractère anxiogène de ces résultats, $V$ not. A. MARAIS, thèse précitée, $\mathrm{n}^{\circ} 27$ et $\mathrm{s}$.

${ }^{64}$ V. déjà en ce sens, P. CATALA, Ebauche d'une théorie juridique de l'information, D. 1984, ch 97 ; Le marché de l'information (aspects juridiques), in Le droit à l'épreuve du numérique, Jus ex machina, PUF, 1998, spéc. p. 336 et s. Il proposait de rattacher les données confidentielles à l'article 9 afin de leur assurer une protection plus efficace. 
individualités" ${ }^{35}$ et a comme finalité d'imposer aux tiers le respect des éléments constitutifs de la personnalité, à créer "une zone de protection" à l'égard des tiers. Attachés à la personne de leur titulaire, sans valeur patrimoniale directe, ils sont intransmissibles, imprescriptibles, insaisissables, mais ils peuvent faire exceptionnellement l'objet de certaines conventions dans un but favorable à l'individu, ce qui serait en cohérence avec l'application proposée du principe de primauté de la personne humaine.

Tendant jusqu'à présent à la protection de l'intégrité physique, de l'intégrité morale de la personne, de sa vie privée ${ }^{67}$, les droits de la personnalité s'affirment comme un réceptacle naturel de la protection de ces données génétiques formant l'identité génétique de la personne. C'est ainsi que pourrait être inscrit au sein du Code civil une disposition s'inspirant à la fois de l'article 16 et de l'article 9 selon laquelle "chacun a droit au respect de son identité génétique" de l'identité génétique qui serait par ailleurs imprescriptible ${ }^{69}$ aurait comme effet d'obliger tout individu, avant d'accomplir toute prestation $^{70}$ en rapport avec une information génétique de la personne, de solliciter le consentement de celle-ci et de s'incliner devant son refus ${ }^{71}$.

Surtout, afin d'assurer une protection maximale de ces information, une autre disposition pourrait s'inspirer des articles 16-1 et 9-1 afin d'autoriser le juge à faire cesser toute atteinte illicite à l'intégrité génétique de la personne par exemple l'utilisation de ses données par un assureur ou un employeur.

${ }^{65}$ E-H Perreau, Des droits de la personnalité, RTDC 1909, 501, spéc. p. 504.

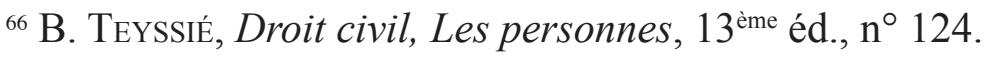

${ }^{67}$ Selon la distinction opérée par F. Terré, D. Fenouillet, Les personnes, la famille, les incapacités, $7^{\text {ème }}$ éd., 2005, $\mathrm{n}^{\circ} 53$.

${ }^{68}$ Cette idée a pour la première fois à notre connaissance été proposée par A. MARAIS sous la forme de la protection de l'intégrité génétique, thèse précitée, $\mathrm{n}^{\circ} 95$ et s., p. 42 et $\mathrm{s}$. Mais le terme identité a été jugé plus conforme à la réalité, le mot intégrité renvoyant à 1' "état d'une chose qui est entière", Littré, $\mathrm{V}^{\circ}$ intégrité. Or ce n'est pas le caractère complet des données génétiques qui doit être protégé mais le fait qu'elles renseignent même isolément sur la personne même. Voir pour une critique du terme "intégrité", F. TERRÉ, D. Fenouillet, op. cit, $\mathrm{n}^{\circ} 67$.

${ }^{69} \mathrm{~V}$. B. TeYssié, op. cit, $\mathrm{n}^{\circ} 127$.

${ }^{70}$ Et pas seulement avant la réalisation de l'examen médical permettant son dévoilement comme le prévoit seulement l'article 16-10 du code civil.

${ }^{71}$ Nous nous inspirons de la réflexion d'A. Marais, thèse précitée, $\mathrm{n}^{\circ} 116, \mathrm{p}$. 49. Le simple droit d'opposition prévu au regard des données de santé dans la loi de 1978 ne paraît pas suffisant. 